\title{
Grand Unified Yukawa Matrix Ansatz: The Standard Model Fermion Mass, Quark Mixing and CP Violation Parameters
}

\author{
Yong-Chao Zhang* and De-Hai Zhang⿵⺆ \\ College of Physical Sciences, Graduate University of Chinese Academy of Sciences, Beijing 100049, P. R. China
}

\begin{abstract}
We propose a new mass matrix ansatz: At the grand unified (GU) scale, the standard model (SM) Yukawa coupling matrix elements are integer powers of the square root of the GU gauge coupling constant $\varepsilon \equiv \sqrt{\alpha_{\mathrm{GU}}}$, multiplied by order unity random complex numbers. It relates the hierarchy of the SM fermion masses and quark mixings to the gauge coupling constants, greatly reducing the $\mathrm{SM}$ parameters, and can give good fitting results of the SM fermion mass, quark mixing and CP violation parameters. This is a neat but very effective ansatz.
\end{abstract}

PACS numbers: $12.10 . \mathrm{Kt}, 12.15 . \mathrm{Ff}, 11.10 . \mathrm{Hi}$

In the standard model (SM), the smallness of the quark and charged lepton masses and the quark mixing angles, and the hierarchy among them has been a problem for decades of years. Altogether with the $\mathrm{CP}$ violation phase, they constitute 13 of all the 19 free parameters in the SM. These 13 parameters may have a common origin at extremely high energy scales and open us a narrow window to the underlying theory beyond the SM. The latest experimental values of the SM fermion masses [1] can be translated to the running masses at $M_{Z}$ with the routine and method of Ref. 2], wich is given in Table I The three quark mixing angles and the $\mathrm{CP}$ violation phase can also be easily obtained from the latest experimental values of the four Wolfenstein parameters of the CKM matrix $[1,3,4]$ :

$$
\begin{aligned}
\sin \theta_{12} & =0.2257_{-0.0010}^{+0.0009}, \\
\sin \theta_{23} & =0.0415_{-0.0015}^{+0.0014}, \\
\sin \theta_{13} & =0.00359_{-0.00034}^{+0.00040}, \\
|\sin \delta| & =0.9329_{-0.0382}^{+0.0178} .
\end{aligned}
$$

In the SM, the neutrinos are definitely massless.

Stitching proper mass matrices is an effective way to reduce the SM parameters, explore the fundamental physics beyond the SM and give meaningful predictions, and many different structures of mass matrices have been

TABLE I: The SM fermion running masses at $M_{Z}$.

\begin{tabular}{ccc}
\hline \hline$m_{u}(\mathrm{MeV})$ & $m_{d}(\mathrm{MeV})$ & $m_{s}(\mathrm{MeV})$ \\
\hline $1.48_{-0.62}^{+0.47}$ & $2.92_{-0.93}^{+0.62}$ & $60_{-21}^{+17}$ \\
\hline \hline$m_{c}(\mathrm{GeV})$ & $m_{b}(\mathrm{GeV})$ & $m_{t}(\mathrm{GeV})$ \\
\hline $0.63_{-0.07}^{+0.06}$ & $2.89_{-0.08}^{+0.15}$ & $170.1 \pm 2.5$ \\
\hline \hline$m_{e}(\mathrm{MeV})$ & $m_{\mu}(\mathrm{MeV})$ & $m_{\tau}(\mathrm{GeV})$ \\
\hline $0.490_{0.000}^{0.000}$ & $103_{0}^{0}$ & $1.746_{0.000}^{0.000}$ \\
\hline \hline
\end{tabular}

*Electronic address: zhangyongch207@mails.gucas.ac.cn

${ }^{\dagger}$ Electronic address: dhzhang@gucas.ac.cn proposed and researched intensively [5-9]. What's more, many relations among the 13 parameters are always incorporated within the fermion mass matrices [8-10], such as the GU bottom-tauon mass unification $m_{b}=m_{\tau}$ and the formula for the Cabibbo angle $\tan ^{2} \theta_{C} \approx m_{d} / m_{s}$.

If all the Yukawa matrix elements are of order unity at extreme high energy scales, e.g. at the GU scale $M_{\mathrm{GU}}$, it is impossible to generate the hierarchical SM fermion spectroscopy naturally, either with the SM or the supersymmetric (SUSY) renormalization group equations (RGEs) [5]. Thus the mass matrices must be constructed hierarchically at some high energy scale.

There is an extremely natural small parameter at hand to construct the hierarchical mass matrices: $\alpha_{\mathrm{GU}} \simeq$ $0.04=0.2^{2}$. It is more convenient to use $\varepsilon \equiv \sqrt{\alpha_{\mathrm{GU}}} \simeq 0.2$ instead of $\alpha_{\mathrm{GU}}$, as 0.04 is too small. We can guess the smallness and hierarchy of the SM fermion masses and the three SM gauge coupling constants have common origin at $M_{\mathrm{GU}}$ : They are related by $\alpha_{\mathrm{GU}} \equiv \varepsilon^{2}$. Coincidentally, $\varepsilon \equiv \sqrt{\alpha_{\mathrm{GU}}} \simeq \lambda$, one parameter in Wolfenstein parameterization of the CKM matrix [3]. We yet stress that the mass matrices are constructed by $\varepsilon \equiv \sqrt{\alpha_{\mathrm{GU}}}$ but not by $\lambda$, which, reflecting the hierarchy of the quark mixings, can be deduced from proper hierarchical quark matrices.

For example, at $M_{\mathrm{GU}}$,

$$
Y_{U}\left(M_{\mathrm{GU}}\right) \sim\left(\begin{array}{lll}
\varepsilon^{n_{u}} & \varepsilon^{n_{u c}} & \varepsilon^{n_{u t}} \\
\varepsilon^{n_{c u}} & \varepsilon^{n_{c}} & \varepsilon^{n_{c t}} \\
\varepsilon^{n_{t u}} & \varepsilon^{n_{t c}} & \varepsilon^{n_{t}}
\end{array}\right),
$$

where $n_{u, c, t}, n_{u c, c u, u t, t u, c t, t c}$ are all non-negative real numbers. The elements must be multiplied by phase factors to generate $\mathrm{CP}$ violation, it is a more natural choice to multiply order unity random complex numbers [5, 6, 11 14]. Specifically, we propose the arguments of the complex numbers be distributed evenly, but the logarithms of the magnitudes of the complex numbers be of normal distribution, where the logarithmic operation guarantees that the magnitudes of the complex numbers are positive definite. If all the exponent numbers of the mass matrices are only non-negative real numbers, with no other constraints, they will be viewed as input parameters. Then there will be more input parameters than the 
TABLE II: $M_{\mathrm{GU}}$ and $\alpha_{\mathrm{GU}}$ for different $M_{\mathrm{SUSY}}$.

\begin{tabular}{cccc}
\hline \hline$M_{\mathrm{SUSY}}$ & $M_{t}$ & $1 \mathrm{TeV}$ & $5 \mathrm{TeV}$ \\
\hline$M_{\mathrm{GU}}\left(10^{16} \mathrm{GeV}\right)$ & 1.87 & 1.42 & 1.11 \\
$\alpha_{\mathrm{GU}}^{-1}$ & 24.71 & 25.87 & 26.89 \\
\hline \hline
\end{tabular}

13 SM phenomenological parameters to fit, which is opposite to the initial desire to reduce the SM parameters. Alternatively, if they are all non-negative integers, then these integers can be viewed as factors from fundamental theories, just as the factor 3 in the Georgi-Jarlskog mass relation [8] (if we consider the matrices are constructed by powers of $\alpha_{\mathrm{GU}}$, then the exponent numbers are non-negative half-integers). For example, they can be deduced from F-theory GUTs [11]. It is rational to suppose further that $n_{u c}=n_{c u}, n_{u t}=n_{t u}, n_{c t}=n_{t c}$, etc., leaving only 6 independent non-negative integers for each mass matrix. In this way, the fermion masses, quark mixings and $\mathrm{CP}$ violation, altogether 13 parameters, can be possibly deduced from proper matrix structures of the single parameter $\varepsilon \equiv \sqrt{\alpha_{\mathrm{GU}}}$, and the GU gauge coupling constant $\alpha_{\mathrm{GU}}$ naturally generates the three SM gauge coupling constants. The free parameters in the SM are greatly reduced.

Choi [15] has shown that GUTs with contorted multiplets of fermions are also available, e.g. pairing of quarks and leptons from different generations such as $(u, d) \oplus\left(\nu_{\mu}, \mu\right)$ and pairing of different left- and righthanded fermions such as $\left(\nu_{e}, e\right)_{L} \oplus \mu_{R}$. General and contorted Yukawa matrices may lead to different values of observable quantities. For simplicity, we do not consider matrices with contorted structures.

The goodness of fit $(\mathrm{GoF})$ is defined to be [16]

$$
\chi^{2}=\sum_{i}\left(\ln \frac{q_{i \text { calc }}}{q_{i \text { expt }}}\right)^{2},
$$

where $q_{i}$ calc and $q_{i}$ expt are respectively quantities from theoretical calculations and experimental observations.

Using the minimal supersymmetric standard model (MSSM) RGEs, the Yukawa matrices are evolved from $M_{\mathrm{GU}}$ to the SUSY scale $M_{\mathrm{SUSY}}$. After the SUSY particles decouple, the matrices are evolved further to lower energy scales with the SM RGEs. The one-loop SM and MSSM $\beta$-functions needed can be found in Refs. [17, 18]. At $M_{Z}$, the SM fermion mass, quark mixing and $\mathrm{CP}$ violation parameters are extracted to fit the experimental values. For simplicity, we ignore the possible effects on these SM parameters from the decoupling of the SUSY particles, the running of the $\tan \beta=v_{u} / v_{d}$ and the transition between different renormalization schemes, and assume the SUSY particles all decouple at a common scale: $M_{\mathrm{SUSY}}$ [2, 19]. We will use mainly $M_{\mathrm{SUSY}}=1 \mathrm{TeV}$ in the following calculation. However, $M_{\mathrm{SUSY}}$ has influence on the values of $M_{\mathrm{SUSY}}$ and $\alpha_{\mathrm{GU}}^{-1}$ to some extent, which is obvious in Table II.
To run the matrices below $M_{\mathrm{SUGY}}$, we must first determine $\tan \beta$. If we suppose $y_{t} \sim y_{b} \sim y_{\tau} \sim 1$ at $M_{\mathrm{GU}}$, $\tan \beta$ must be large. To obtain $\tan \beta$ conveniently, the three Yukawa matrices can be approximated

$$
Y_{U, D, E}\left(M_{\mathrm{GU}}\right) \sim\left(\begin{array}{ccc}
0 & 0 & 0 \\
0 & 0 & 0 \\
0 & 0 & 1
\end{array}\right)
$$

with order unity random complex numbers multiplying the $(3,3)$ elements. When the matrices are evolved from $M_{\mathrm{GU}}$ to $M_{Z}$ and the third generation masses are compared to the experimental values given in Table \, we obtain reliably $\tan \beta \approx 55$, ignoring the effect from $M_{\mathrm{SUGY}}$. The value of $\tan \beta$ is consistent with the solution given in Ref. [17].

We find that when all the exponent integers of the three SM Yukawa coupling matrices take proper values at $M_{\mathrm{GU}}$, it is possible to obtain good fitting results of the nine SM fermion masses. For example, when

$$
\begin{aligned}
Y_{U}\left(M_{\mathrm{GU}}\right) & \sim\left(\begin{array}{lll}
\varepsilon^{8} & \varepsilon^{6} & \varepsilon^{4} \\
\varepsilon^{6} & \varepsilon^{4} & \varepsilon^{3} \\
\varepsilon^{4} & \varepsilon^{3} & 1
\end{array}\right), \\
Y_{D}\left(M_{\mathrm{GU}}\right) & \sim\left(\begin{array}{lll}
\varepsilon^{5} & \varepsilon^{4} & \varepsilon^{3} \\
\varepsilon^{4} & \varepsilon^{3} & \varepsilon^{2} \\
\varepsilon^{3} & \varepsilon^{2} & 1
\end{array}\right), \\
Y_{E}\left(M_{\mathrm{GU}}\right) & \sim\left(\begin{array}{lll}
\varepsilon^{5} & \varepsilon^{4} & \varepsilon^{3} \\
\varepsilon^{4} & \varepsilon^{3} & \varepsilon \\
\varepsilon^{3} & \varepsilon & 1
\end{array}\right),
\end{aligned}
$$

we will get at $M_{Z}$

$$
\begin{aligned}
& m_{u}=2.14 \mathrm{MeV}, m_{c}=0.599 \mathrm{GeV}, m_{t}=176.9 \mathrm{GeV}, \\
& m_{d}=3.21 \mathrm{MeV}, m_{s}=55.6 \mathrm{MeV}, m_{b}=3.08 \mathrm{GeV}, \\
& m_{e}=0.951 \mathrm{MeV}, m_{\mu}=136 \mathrm{MeV}, m_{\tau}=1.84 \mathrm{GeV}(.6)
\end{aligned}
$$

Compared with the experimental values in Table I using Eq. (3), we can obtain a small GoF $\chi^{2}=0.68$ for all the nine SM fermion masses. When the experimental errors are taken into consideration, the GoF is even smaller: $\chi^{2}=0.53$.

Matrices with vanishing elements at $M_{\mathrm{GU}}$ are also possible choice [7 9]. The exponent integers of the vanishing elements are infinity. For example, if we take

$$
Y_{U}\left(M_{\mathrm{GU}}\right) \sim\left(\begin{array}{ccc}
\varepsilon^{8} & 0 & \varepsilon^{4} \\
0 & \varepsilon^{4} & 0 \\
\varepsilon^{4} & 0 & 1
\end{array}\right)
$$

the up-type quark masses at $M_{Z}$ are

$$
m_{u}=1.55 \mathrm{MeV}, m_{c}=0.597 \mathrm{GeV}, m_{t}=176.9 \mathrm{GeV}
$$

with a very small GoF $\chi^{2}=7.1 \times 10^{-3}$ for the three masses. Examples of down-type quark and charged lep- 
ton matrices with vanishing elements are as follows:

$$
Y_{D}\left(M_{\mathrm{GU}}\right) \sim\left(\begin{array}{ccc}
0 & \varepsilon^{4} & 0 \\
\varepsilon^{4} & \varepsilon^{3} & \varepsilon^{2} \\
0 & \varepsilon^{2} & 1
\end{array}\right),
$$

with mass eigenvalues at $M_{Z}$

$$
m_{d}=2.21 \mathrm{MeV}, m_{s}=55.4 \mathrm{MeV}, m_{b}=3.08 \mathrm{GeV}
$$

and a very small GoF $\chi^{2}=8.8 \times 10^{-3}$ for the three down-type quark masses;

$$
Y_{E}\left(M_{\mathrm{GU}}\right) \sim\left(\begin{array}{ccc}
0 & \varepsilon^{4} & \varepsilon^{3} \\
\varepsilon^{4} & \varepsilon^{2} & 0 \\
\varepsilon^{3} & 0 & 1
\end{array}\right),
$$

with mass eigenvalues at $M_{Z}$

$$
m_{e}=0.337 \mathrm{MeV}, m_{\mu}=125 \mathrm{MeV}, m_{\tau}=1.77 \mathrm{GeV}
$$

and a small GoF $\chi^{2}=0.18$ for the three charged leptons masses. There are no constraints on the structures of the mass matrices; in principle we can construct any textures that lead to well fitted fermion masses. We can even construct matrices at $M_{\mathrm{GU}}$, whose first and second family fermion masses are both completely from mixing with other generations. Here is an example:

$$
Y_{U}\left(M_{\mathrm{GU}}\right) \sim\left(\begin{array}{ccc}
0 & \varepsilon^{6} & \varepsilon^{5} \\
\varepsilon^{6} & 0 & \varepsilon^{2} \\
\varepsilon^{5} & \varepsilon^{2} & 1
\end{array}\right) .
$$

Its mass eigenvalues at $M_{Z}$ are

$$
m_{u}=1.38 \mathrm{MeV}, m_{c}=0.821 \mathrm{GeV}, m_{t}=176.3 \mathrm{GeV}
$$

with a very small GoF $\chi^{2}=0.073$ for the three masses.

It is worth noting that $M_{\mathrm{SUSY}}$ has considerable effect on the SM fermion spectroscopy. All the masses above are obtained with $M_{\mathrm{SUSY}}=1 \mathrm{TeV}$. For the matrices in Eq. (5), if we take $M_{\mathrm{SUSY}}=M_{t}$,

$$
\begin{aligned}
& m_{u}=2.62 \mathrm{MeV}, m_{c}=0.673 \mathrm{GeV}, m_{t}=173.8 \mathrm{GeV} ; \\
& \text { if } M_{\mathrm{SUSY}}=5 \mathrm{TeV}, \\
& m_{u}=1.80 \mathrm{MeV}, m_{c}=0.543 \mathrm{GeV}, m_{t}=179.3 \mathrm{GeV} .
\end{aligned}
$$

Compared the up-type quark mass eigenvalues with different $M_{\mathrm{SUSY}}$, the effect on the first and second generation masses is obvious. This is a general phenomenology for the quark and charged lepton masses, whatever structure the fermion mass matrices are at $M_{\mathrm{GU}}$.

To obtain well fitted quark mixing and $\mathrm{CP}$ violation parameters, we must pare suitable up- and down-type quark matrices. Not all paring of good fitting up- and down-type quark matrices lead to well fitted quark mixings and $\mathrm{CP}$ violation, such as the first two matrices of
Eq. (5) which would give much larger $\sin \theta_{13}=0.0136$ than the experimental value $\sin \theta_{13}=0.00359$. When we choose

$$
\begin{aligned}
& Y_{U}\left(M_{\mathrm{GU}}\right) \sim\left(\begin{array}{lll}
\varepsilon^{8} & \varepsilon^{6} & \varepsilon^{4} \\
\varepsilon^{6} & \varepsilon^{4} & \varepsilon^{3} \\
\varepsilon^{4} & \varepsilon^{3} & 1
\end{array}\right), \\
& Y_{D}\left(M_{\mathrm{GU}}\right) \sim\left(\begin{array}{lll}
\varepsilon^{5} & \varepsilon^{4} & \varepsilon^{4} \\
\varepsilon^{4} & \varepsilon^{3} & \varepsilon^{3} \\
\varepsilon^{4} & \varepsilon^{3} & 1
\end{array}\right),
\end{aligned}
$$

we get at $M_{Z}$

$$
\begin{aligned}
& \sin \theta_{12}=0.227, \sin \theta_{23}=0.0189, \sin \theta_{13}=0.00402, \\
& |\sin \delta|=0.651
\end{aligned}
$$

with a GoF $\chi^{2}=0.76$ for the four parameters. Quark matrices with vanishing elements are also available. For example,

$$
\begin{aligned}
Y_{U}\left(M_{\mathrm{GU}}\right) & \sim\left(\begin{array}{ccc}
0 & 0 & \varepsilon^{4} \\
0 & \varepsilon^{4} & \varepsilon^{3} \\
\varepsilon^{4} & \varepsilon^{3} & 1
\end{array}\right), \\
Y_{D}\left(M_{\mathrm{GU}}\right) & \sim\left(\begin{array}{ccc}
0 & \varepsilon^{4} & 0 \\
\varepsilon^{4} & \varepsilon^{3} & \varepsilon^{3} \\
0 & \varepsilon^{3} & 1
\end{array}\right)
\end{aligned}
$$

lead to

$$
\begin{aligned}
& \sin \theta_{12}=0.219, \sin \theta_{23}=0.0186, \sin \theta_{13}=0.00257, \\
& |\sin \delta|=0.649
\end{aligned}
$$

at $M_{Z}$ with a GoF $\chi^{2}=0.89$ for the four parameters. All the mass eigenvalues of the quark matrices in Eq. (13) and Eq. (15) are well consistent with experiments.

$M_{\text {SUSY }}$ also has possible effect on the quark mixing and $\mathrm{CP}$ violation parameters. For the quark matrices in Eq. (13), if $M_{\mathrm{SUSY}}=M_{t}$,

$$
\begin{aligned}
& \sin \theta_{12}=0.231, \sin \theta_{23}=0.0211, \sin \theta_{13}=0.00458 \\
& |\sin \delta|=0.651
\end{aligned}
$$

with a $\operatorname{GoF} \chi^{2}=0.65$ for the four parameters; if $M_{\mathrm{SUSY}}=5 \mathrm{TeV}$,

$$
\begin{aligned}
& \sin \theta_{12}=0.223, \sin \theta_{23}=0.0173, \sin \theta_{13}=0.00359 \\
& |\sin \delta|=0.651
\end{aligned}
$$

with a GoF $\chi^{2}=0.90$ for the four parameters. Compared the four parameters in Eq. (16), (14) and (17), the effect of $M_{\text {SUSY }}$ on $\sin \theta_{12}$ and $\sin \theta_{23}$ is small, the effect on $\sin \theta_{13}$ is comparatively large, but $|\sin \delta|$ is hardly subject to $M_{\mathrm{SUSY}}$. It is almost the same when the quark matrices are of other structures. The hierarchical structure of the CKM matrix, which is reflected by the powers of $\lambda$ in the Wolfenstein parameterization, can obtain naturally from proper quark matrices, as we stated above. 
In conclusion, we have proposed a new ansatz for the SM fermion mass and mixing generation: At $M_{\mathrm{GU}}$ the Yukawa coupling matrices are constructed by nonnegative integer powers of $\varepsilon \equiv \sqrt{\alpha_{\mathrm{GU}}}$, i.e. by nonnegative half-integer powers of $\alpha_{\mathrm{GU}}$, with matrix elements multiplied by order unity random complex numbers. By all the examples given above, we have demon- strated that this is a neat but extremely effective ansatz: It not only relates the hierarchy of SM fermion masses and quark mixings to the gauge coupling constants, reducing the number of the SM parameters to the utmost extent, but can give well fitted SM fermion mass, quark mixing and $\mathrm{CP}$ violation parameters. It could also apply to the neutrino mass and oscillation problem.
[1] C. Amsler et al. (Particle Data Group), Phys. Lett. B 667, 1(2008).

[2] Z. Z. Xing, H. Zhang, and S. Zhou, Phys. Rev. D 77, 113016 (2008) arXiv:0712.1419 [hep-ph]].

[3] L. Wolfenstein, Phys. Rev. Lett. 51,1945 (1983).

[4] N. Cabibbo, Phys. Rev. Lett. 10, 531 (1963); M. Kobayashi and T. Maskawa, Prog. Theor. Phys. 49, 652 (1973).

[5] C. D. Froggatt and H. B. Nielsen, Nucl. Phys. B 147, 277 (1979).

[6] C. D. Froggatt and H. B. Nielsen, Nucl. Phys. B 164, 114 (1979); Surveys High Energ. Phys. 18, 55 (2003) arXiv:hep-ph/0308144.

[7] S. Dimopoulos, L. J. Hall, and S. Raby, Phys. Rev. D 45, 4192 (1992); Phys. Rev. Lett. 68, 1984 (1992);

G. F. Giudice, Mod. Phys. Lett. A 7, 2429 (1992) arXiv:hep-ph/9204215;

K. S. Babu and Q. Shafi, arXiv:hep-ph/9209214

P. Ramond, R. G. Roberts, and G. G. Ross, Nucl. Phys. B 406, 19 (1993) arXiv:hep-ph/9303320;

M. Leurer, Y. Nir, and N. Seiberg, Nucl. Phys. B 420, 468 (1994) arXiv:hep-ph/9310320;

L. E. Ibanez and G. G. Ross, Phys. Lett. B 332, 100 (1994) arXiv:hep-ph/9403338;

R. Barbieri, G. Dvali, and L. Hall, Phys. Lett. B 377, 76 (1996) arXiv:hep-ph/9512388;

E. J. Chun and A. Lukas, Phys. Lett. B 387, 99 (1996) arXiv:hep-ph/9605377;

J. L. Chkareuli and C. D. Froggatt, Phys. Lett. B 450, 158 (1999) arXiv:hep-ph/9812499;

H. Fritzsch and Z. Z. Xing, Prog. Part. Ncul. Phys. 45, 1 (2000) arXiv:hep-ph/9912358;

J. L. Chkareuli, C. D. Froggatt, and H. B. Nielsen, Nucl. Phys. B 626, 307 (2002) arXiv:hep-ph/0109156.

[8] H. Georgi and C. Jarlskog, Phys. Lett. B 86, 297 (1979).

[9] H. Fritzsch, Phys. Lett. B 70, 436 (1977); ibid. 73, 317 (1978); Nucl. Phys. B 155, 189 (1979);

T. Kitazoe and K. Tanaka, Phys. Rev. D 18, 3476 (1978); J. A. Harvey, P. Ramond, and D. B. Reiss, Phys. Lett. B 92, 309 (1980); Nucl. Phys. B 199, 223 (1982).

[10] R. Gatto, G. Sartori, and M. Tonin, Phys. Lett. B 28,
128 (1968);

R. J. Oakes, Phys. Lett. B 29, 683 (1969);

M. S. Chanowitz, J. R. Ellis, and M. K. Gaillard, Nucl. Phys. B 128, 506 (1977);

F. Wilczek and Z. Zee, Phys. Lett. B 70, 418 (1977);

S. Weinberg, Trans. New York Acad. Sci. 38, 185 (1977); A. De Rújula, H. Georgi, and S. L. Glashow, Ann. Phys. 109, 258 (1977);

A. J. Buras, J. R. Ellis, M. K. Gaillard, and D. V. Nanopoulos, Nucl. Phys. B 135, 66 (1978);

T. Hagiwara, T. Kitazoe, G. B. Mainland, and K. Tanaka, Phys. Lett. B 76, 602 (1978);

H. Arason, D. J. Castaño, E. J. Piard, and P. Ramond, Phys. Rev. D 47, 232 (1993) arXiv:hep-ph/9204225; L. J. Hall and A. Rašin, Phys. Lett. B 315, 164 (1993) arXiv:hep-ph/9303303.

[11] J. J. Heckman and C. Vafa, arXiv:0811.2417 [hep-th]; J. J. Heckman and C. Vafa, arXiv:0904.3101 [hep-th]; S. Cecotti, M. C. N. Cheng, J. J. Heckman, and C. Vafa, arXiv:0910.0477 [hep-th].

[12] G. W. Gibbons, S. Gielen, C. N. Pope, and N. Turok, Phys. Rev. Lett. 102, 121802 (2009) arXiv:0810.4368 [hep-th]].

[13] L. J. Hall, M. P. Salem, and T. Watari, Phys. Rev. D 76, 093001 (2007) arXiv:0707.3446 [hep-ph]].

[14] A. E. Nelson and M. J. Strassler, Journal of High Energy Physics 0009, 030 (2000) arXiv:hep-ph/0006251.

[15] K. S. Choi, Phys. Lett. B 668, 392 (2008) arXiv:0807.2766 [hep-ph]].

[16] C. D. Froggatt, H. B. Nielsen, and D. J. Smith, Phys. Lett. B 385, 150 (1996) arXiv:hep-ph/9607250.

[17] V. D. Barger, M. S. Berger, and P. Ohmann, Phys. Rev. D 47, 1093 (1993) arXiv:hep-ph/9209232.

[18] H. Arason, D. J. Castaño, B. Kesthelyi, S. Mikaelian, E. J. Piard, P. Ramond, and B. D. Wright, Phys. Rev. D 46, 3945 (1992);

D. J. Castaño, E. J. Piard, and P. Ramond, ibid. 49, 4882 (1994) arXiv:hep-ph/9308335.

[19] S. Antusch and M. Spinrath, Phys. Rev. D 78, 075020 (2008) arXiv:0804.0717 [hep-ph]]. 